\title{
The Biphasic Root Growth Response to Abscisic Acid in Arabidopsis Involves Interaction with Ethylene and Auxin Signalling Pathways
}

\author{
Xiaoqing Li*, Lin Chen*, Brian G. Forde and William J. Davies \\ Lancaster Environment Centre, Lancaster University, Lancaster, United Kingdom
}

OPEN ACCESS

Edited by:

Dominique Van Der Straeten,

Ghent University, Belgium

Reviewed by:

Chi-Kuang Wen,

Shanghai Institutes for Biological

Sciences (CAS), China

Nihal Dharmasiri,

Texas State University, United States

*Correspondence:

Lin Chen

lin.chen@york.ac.uk;

ynclily@hotmail.com

Xiaoqing $\mathrm{Li}$

lixqymail@gmail.com

${ }^{\dagger}$ Present address:

Xiaoqing $\mathrm{Li}$,

CSIRO Agriculture and Food

Canberra, ACT, Australia

Lin Chen,

Department of Biology, University of

York, York, United Kingdom

Specialty section:

This article was submitted to

Plant Physiology,

a section of the journal

Frontiers in Plant Science

Received: 01 March 2017

Accepted: 11 August 2017

Published: 25 August 2017

Citation:

Li X, Chen L, Forde BG and Davies WJ (2017) The Biphasic Root Growth Response to Abscisic Acid in Arabidopsis Involves Interaction with Ethylene and Auxin Signalling Pathways. Front. Plant Sci. 8:1493.

doi: 10.3389/fpls.2017.01493
Exogenous abscisic acid (ABA) is known to either stimulate or inhibit root growth, depending on its concentration. In this study, the roles of ethylene and auxin in this biphasic effect of ABA on root elongation were investigated using chemical inhibitors and mutants. Inhibitors of ethylene perception and biosynthesis and an auxin influx inhibitor were all found to block the inhibitory effect of high ABA concentrations, but not the stimulatory effect of low ABA concentrations. In addition, three ethylene-insensitive mutants (etr1-1, ein2-1, and ein3-1), two auxin influx mutants (aux1-7, aux1-T) and an auxin-insensitive mutant (iaa7/axr2-1) were all insensitive to the inhibitory effect of high ABA concentrations. In the case of the stimulatory effect of low ABA concentrations, it was blocked by two different auxin efflux inhibitors and was less pronounced in an auxin efflux mutant (pin2/eir1-1) and in the iaa7/axr2-1 auxin-insensitive mutant. Thus it appears that the stimulatory effect seen at low ABA concentrations is via an ethyleneindependent pathway requiring auxin signalling and auxin efflux through PIN2/EIR1, while the inhibitory effect at high ABA concentrations is via an ethylene-dependent pathway requiring auxin signalling and auxin influx through AUX1.

\footnotetext{
Keywords: abscisic acid (ABA), Arabidopsis, auxin transport, auxin signalling, ethylene biosynthesis, ethylene signalling, hormone, root elongation
}

\section{INTRODUCTION}

Plant growth and yield production are often limited by a variety of abiotic stresses in agricultural systems (Cramer et al., 2011). A root system that is able to efficiently take up water and nutrient from the soil is crucial for plant growth and functioning, particularly if the plant is to accumulate any yield when water and nutrients are in short supply (Hammer et al., 2009; Hodge et al., 2009; Hodge, 2010). Previous studies have reported that mild soil drying stimulates root growth, but when soil drying becomes more severe, it inhibits root growth (Sharp and Davies, 1979; Watts et al., 1981). However, there is no consensus on the mechanisms underlying these root responses. An improved understanding of the mechanistic basis of root growth and development will be useful

Abbreviations: ABA, abscisic acid; [ABA], abscisic acid concentration; ACC, 1-aminocyclopropane-1-carboxylic acid; $\mathrm{Ag}^{+}$, silver ion; ARF, auxin response factor; AUX/IAA, auxin/indole-3-acetic acid; AUX1, AUXIN 1 (auxin influx transporter); AVG, aminoethoxyvinylglycine (ethylene biosynthesis inhibitor); CHPAA, 3-chloro-4-hydroxyphenylacetic acid (auxin influx inhibitor); GFP, green fluorescent protein; NPA, $N$-1-naphthylphthalamidic acid (auxin efflux inhibitor); PIN, PINFORMED (auxin efflux transporter); STS, silver thiosulfate (ethylene signalling inhibitor); TIBA, 2,3,5-triiodobenzoic acid (auxin efflux inhibitor). 
both for novel crop management and breeding aimed at plant improvement to at least maintain yield in different environments under a changing climate.

Plant hormones are crucial regulators of plant growth and development (Davies, 2010). Among them, abscisic acid (ABA) has been recognised as a stress-hormone and its regulation of plant drought responses has been extensively studied (e.g., Hsiao, 1973; Schachtman and Goodger, 2008; Cutler et al., 2010). ABA accumulates under soil drying and the endogenous ABA concentration in the plant can be an indicator of soil water availability (Zhang and Davies, 1989). Generally, ABA is known as an inhibitor of shoot and root growth of plants under wellwatered conditions (Sharp et al., 1994; Sharp and LeNoble, 2002) and previous studies have shown that ABA acts as an inhibitor of growth of plants under water deficit (Bensen et al., 1988; Creelman et al., 1990; Rowe et al., 2016). On the other hand, maize plants with reduced endogenous ABA content (by genetic modification or chemical treatments) had roots that were more sensitive to the inhibitory effect of low water potential, indicating that $\mathrm{ABA}$ plays a role in maintaining root elongation under low water potentials (Saab et al., 1990). However, other studies have indicated complex biphasic effects of exogenous $\mathrm{ABA}$ on root growth under well-watered conditions, where relatively low concentrations of ABA stimulated root growth while high concentrations inhibited root growth (Watts et al., 1981; Xu et al., 2013). This is analogous to the biphasic effects of soil drying on root growth where mild water deficit stimulated root growth while more severe water deficit inhibited root growth (Sharp and Davies, 1979; Watts et al., 1981; Creelman et al., 1990).

Ethylene is another major hormone that mediates plant responses to abiotic stresses, including water deficit (Morgan and Drew, 1997; Spollen et al., 2000; Davies, 2010). Ethylene and its precursor 1-aminocyclopropane-1-carboxylic acid (ACC) have been reported to inhibit root cell elongation, thus inhibiting root growth (Le et al., 2001; Růžička et al., 2007). Alarcón et al. (2009) found that root elongation rate in maize decreased as ethylene production increased. The involvement of ethylene in ABA-regulated root growth was investigated in further detail by Beaudoin et al. (2000) and Ghassemian et al. (2000) who found that root growth in a number of ethylene signalling mutants was less sensitive to ABA $(1-150 \mu \mathrm{M})$ which caused inhibition on root growth, but that the ethylene biosynthesis inhibitor aminoethoxyvinylglycine (AVG) even enhanced sensitivity to the inhibition of root growth caused by ABA (Ghassemian et al., 2000). These results indicated ethylene signalling plays a positive role in inhibiting root growth under high $\mathrm{ABA}$ concentration, but this inhibition does not necessarily involve de novo ethylene biosynthesis. In partial contradiction, a recent study found that ethylene biosynthesis is necessary for the inhibitory effect of high ABA concentration on root growth (Luo et al., 2014). To our knowledge, a role for ethylene in the stimulatory effect of low ABA concentrations on root growth has not been explored.

The hormone auxin is generally recognised as a master regulator in plant root development (Saini et al., 2013). Studies using mutants and protein analysis have provided evidence for crosstalk between auxin and ABA signalling pathways in the root (Bianchi et al., 2002; Rock and Sun, 2005). Mutants that are resistant to both auxin and $\mathrm{ABA}$ (e.g., axr2) also provided genetic evidence for the interaction between auxin and ABA signalling pathways (Pickett et al., 1990; Wilson et al., 1990; Tian and Reed, 1999). However, the external ABA concentrations applied in these earlier studies were relatively high $(1-150 \mu \mathrm{M})$, based on previous estimates of the ABA concentration in wellwatered Arabidopsis root tips as $100 \mathrm{ng} \mathrm{g}^{-1} \mathrm{FW}$ (Xu et al., 2013) (corresponding to a tissue concentration of approximately $0.5 \mu \mathrm{M})$. Lower ABA concentrations $(0.1 \mu \mathrm{M})$ were used in a recent study which reported a role for $\mathrm{ABA}$ in modulating auxin transport in the Arabidopsis root apex to maintain root growth under moderate water stress (Xu et al., 2013). However, the role of the auxin signalling pathway in the response to low $\mathrm{ABA}$ concentrations has not yet been examined. In addition, how auxin transport could involve in root responses to ABA, especially high ABA concentrations, is less clear.

Tissue ABA concentrations can gradually increase to more than 30 times that in the well-watered plants as soil water content slowly decreases (Zhang and Davies, 1989), or up to 10 times that in non-stressed plants when plants were subjected to salt stress (up to $300 \mathrm{mM} \mathrm{NaCl}$ ) (Jia et al., 2002). Therefore, investigating the effects of external application of both low and high concentrations of ABA on plant root growth, and the involvement of other hormones, i.e., auxin and ethylene, can improve our understanding of how plants respond to different levels of stress (e.g., water stress). Such understanding may then facilitate research aimed at enhancing plant performance under those abiotic stresses. Here, we hypothesise that the biphasic effect of low and high ABA concentrations on root growth involves auxin and ethylene. Five chemical inhibitors and 12 mutant lines that are relevant to ethylene and auxin signalling were used to test this hypothesis.

\section{MATERIALS AND METHODS}

\section{Plant Materials}

The wild-type accession of Arabidopsis thaliana L. used in this study was Col-8 (European Arabidopsis Stock Centre catalogue no. N60000). Besides, the auxin influx AUX1 mutants aux1-T (N657534), aux1-7 (N9583); the auxin efflux mutants pin2/eir1-1 (N8058), pin3-4 (N9363), pin3-5 (N9364), pin4-3 (N9368), and pin7-2 (N9366); and auxin signalling mutants iaa7/axr2-1 (N3077) and tir1-1 (N3798) were obtained from the European Arabidopsis Stock Centre. The ethylene-insensitive mutants etr1-1 (ETHYLENE RESPONSE 1) (Bleecker et al., 1988), ein2-1 (ETHYLENE INSENSITIVE 2) (Guzmán and Ecker, 1990), and ein3-1 (Roman et al., 1995) were kindly provided by Dr. Mike Roberts (Lancaster University, United Kingdom). The auxin reporter line DR5::GFP (Ottenschläger et al., 2003) was a kind gift from Prof. Klaus Palme (University of Freiburg, Germany). All Arabidopsis lines were in the Columbia background.

Surface-sterilised seeds were sown on solid medium containing 0.02 x B5 medium, $1 \mathrm{mM} \mathrm{KNO}_{3}, 0.5 \%$ (w/v) sucrose and $1 \%$ agar in $90 \mathrm{~mm}$ diameter Petri dishes (Zhang and Forde, 1998). After stratifying the seed in the dark $\left(4^{\circ} \mathrm{C}\right)$ for $2-3$ days, the Petri dishes were incubated in a vertical orientation in a 
growth room at $22^{\circ} \mathrm{C}$ with a $16 \mathrm{~h}$ light period and an irradiance of $100 \mu \mathrm{mol} \mathrm{m}^{-2} \mathrm{~s}^{-1}$. Four to five days later, seedlings with similar root length were transferred to fresh plates containing ABA at different concentrations. Five inhibitors were added to the growth medium as required: namely, the ethylene biosynthesis inhibitor AVG (0.3 or $0.5 \mu \mathrm{M})$ (A6685, Sigma-Aldrich); the ethylene perception inhibitor silver thiosulfate (STS, $10 \mu \mathrm{M}$ ); and the auxin efflux inhibitors $N$-1-naphthylphthalamidic acid (NPA, $10 \mu \mathrm{M}$ ) (PS343, Sigma-Aldrich), 2,3,5-triiodobenzoic acid (TIBA, $10 \mu \mathrm{M}$ ) (T5910, Sigma-Aldrich); and the auxin influx inhibitor 3-chloro-4-hydroxyphenylacetic acid (CHPAA, $10 \mu \mathrm{M})(224529$, Sigma-Aldrich). For plates with treatments, 3-6 seedlings were placed on $9 \mathrm{~cm}$ diameter plates $(25 \mathrm{ml}$ medium), or 7-9 seedlings on $12 \mathrm{~cm}$ square plates (50 ml medium). The top one-fifth of the agar medium was excised so that the shoot was not in direct contact with the medium. ABA (A1296, SigmaAldrich) stock solutions were made in $10 \mathrm{mM}$ (+ABA) with 0.03 $\mathrm{M} \mathrm{KOH}$. A $60 \mathrm{mM}$ STS solution was freshly prepared by mixing $300 \mathrm{mM}$ silver nitrate with $300 \mathrm{mM}$ sodium thiosulphate in a 1:4 $(\mathrm{v} / \mathrm{v})$ ratio.

\section{Root Growth Measurements}

Primary root growth was monitored during the 3-6 days after seedlings were transferred to the treatment plates by marking the position of the root tips on the base of the plate at 24 or $48 \mathrm{~h}$ intervals. At the end of each experiment, the plates were imaged on a flat-bed scanner with a ruler as the reference. The images were semi-manually analysed using Optimas Image Analysis software (Version 6.1 Media Cybernetics, Inc., United States) for root length. The primary root lengths at the beginning and the end of an experiment were presented in Supplementary Table 1.

\section{Confocal Microscopy}

After 3 days of ABA treatments, DR5::GFP seedlings were stained briefly $(50 \mathrm{~s})$ with $10 \mu \mathrm{M}$ propidium iodide. GFP and propidium iodide fluorescence was then detected using a Leica SP2-AOBS confocal laser scanning microscope and the images were electronically superimposed using LCS Lite software (Leica, Germany). Quantification of the GFP fluorescence signal was performed using ImageJ (National Institutes of Health, United States).

\section{Statistical Analysis}

The statistical software SPSS 21.0 (IBM, United States) was used to perform one-way or two-way ANOVA with Tukey's post hoc test at the $P<0.05$ level. The effect size of those ANOVA was reported by eta ${ }^{2}$ or partial $\mathrm{eta}^{2}$. The criteria for effect size: no effect, eta ${ }^{2}=0$; small, eta ${ }^{2}=0.0099$; medium, eta ${ }^{2}=0.0588$; large, eta $^{2}=0.1379$ (Richardson, 2011).

\section{RESULTS}

\section{Effect of Exogenous ABA on Root Growth}

A detailed comparison of the effects of a range of $\mathrm{ABA}$ concentrations on root elongation was performed by transferring
4 day-old Arabidopsis seedlings to vertical agar plates containing 0 (control), $0.1,1$, and $10 \mu \mathrm{M} \mathrm{ABA}$ and measuring the increase in root length at daily intervals over the following 6 days (Figure 1). The results showed that $10 \mu \mathrm{M}$ ABA inhibited root growth by about $40 \%$ while $0.1 \mu \mathrm{M}$ ABA stimulated growth by almost $20 \%$ when measured over the 6-day period (Figure 1A). The stimulatory effect of $0.1 \mu \mathrm{M}$ ABA persisted over the duration of the treatment and by the 6th day the roots were growing at a rate which was more than $30 \%$ faster than the control (Figure 1B). It appears that the intermediate concentration of ABA used $(1 \mu \mathrm{M})$ is close to the threshold for the transition from stimulation to inhibition as it had little effect on root elongation (Figures 1A,B). In subsequent experiments, concentrations less than $1 \mu \mathrm{M}$ ABA (usually $0.1 \mu \mathrm{M}$ ABA) were therefore used for studying the stimulatory effect of low ABA concentrations and concentrations greater than $1 \mu \mathrm{M}$ ABA (usually $10 \mu \mathrm{M}$ ABA) were used for studying the inhibitory effect of high ABA concentrations.

\section{Investigating the Role of Ethylene in the Root Responses to High and Low Concentrations of ABA}

It has previously been established that the inhibitory effect of high ABA concentrations on root growth is an ethylene-dependent process (Ghassemian et al., 2000). To confirm these findings under our experimental conditions and to investigate whether the stimulatory effect of low ABA concentrations is also ethylenedependent, seedlings were treated with different concentrations of $\mathrm{ABA}$ in the presence or absence of either AVG (an ethylene biosynthesis inhibitor) or STS (an ethylene perception inhibitor). The primary root elongation rates were determined over a 4-day period of treatment. When 0.3 or $0.5 \mu \mathrm{M}$ AVG was included along with the $10 \mu \mathrm{M}$ ABA treatment, the inhibitory effect was relieved as measured after either 1 day (Figure $2 \mathbf{A}$ ) or 4 days (Figure 2B).

In contrast to AVG's ability to interfere with the inhibitory effect of ABA, the presence of either 0.3 or $0.5 \mu \mathrm{M}$ AVG had no influence on the trend of the stimulatory effect after adding $0.1 \mu \mathrm{M}$ ABA (Figures 2A,B). In addition, AVG treatment tended to promote root growth which might mask the stimulatory effect of ABA at low concentrations (Figures 2A,B and data not shown). Thus while ethylene biosynthesis is required for the inhibitory effect of high ABA concentrations it is not required for the stimulatory effect of low ABA concentrations.

When $10 \mu \mathrm{M}$ STS was used to interfere with ethylene perception it almost completely overcame the inhibitory effect of $10 \mu \mathrm{M}$ ABA when measured after the first 2 days of treatment (Figure 2C). This antagonistic effect was lost when root growth was measured over a 4-day period (Figure 2D), which we attribute to the known instability of STS $\left(\mathrm{Ag}^{+}\right)$when exposed to light. However, when included along with $0.1 \mu \mathrm{M} A B A$, the STS did not interfere with the stimulatory effect on root growth as measured after either 2 or 4 days (Figures 2C,D). Therefore, the inhibitory effect of high ABA concentrations, but not the stimulatory effect of low ABA concentrations, could be eliminated by interfering with ethylene perception. 

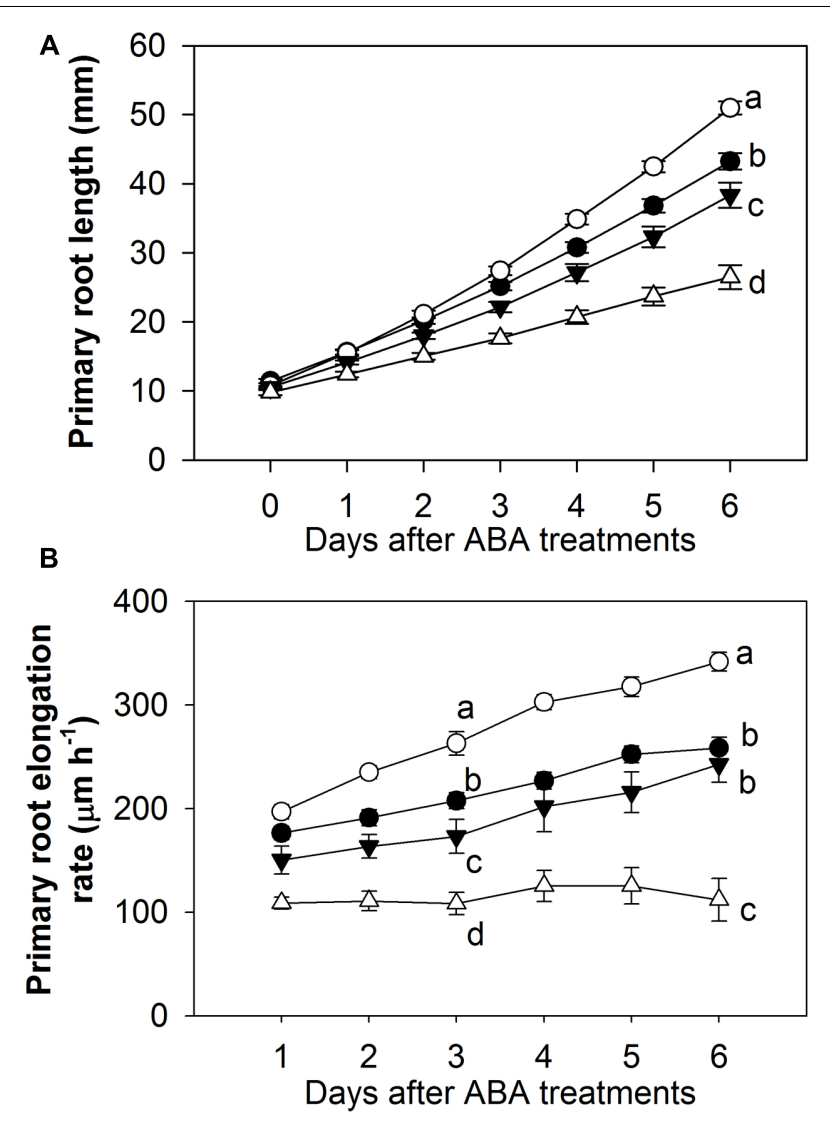

FIGURE 1 | Biphasic effect of applied exogenous ABA on the growth of primary root over the 6-day treatments. (A) Total primary root length. (B) Primary root elongation rate. Four-day old Arabidopsis wild-type Col-8 seedlings with similar root length were chosen and transferred to newly made $0.02 \times \mathrm{B} 5$ medium ( $1 \mathrm{mM} \mathrm{KNO}_{3}, 0.5 \%$ sucrose) with various ABA concentrations (black circle, control; white circle, $0.1 \mu \mathrm{M} A B A$; black triangle, $1 \mu \mathrm{M}$ ABA; white triangle, $10 \mu \mathrm{M}$ ABA). Primary root length was marked after transplanting and the increase of primary root were measured every day. The root elongation rate was calculated for each day. The values are means, and the vertical bars represent standard errors. Data analysed using one-way ANOVA with Tukey's post hoc test and different letters indicate significant differences among ABA treatments in the same day at $P<0.05 . \mathrm{Eta}^{2}$ of one-way ANOVA: (A) 0.776 (day 6); (B) 0.694 (day 3); 0.737 (day 6). Seedling numbers: control, $n=14$; $0.1 \mu \mathrm{M} \mathrm{ABA}, n=9-14$; $1 \mu \mathrm{M} \mathrm{ABA}, n=10-14$; $10 \mu \mathrm{M}$ ABA, $n=11-14$. At least three independent experiments were performed and similar results obtained and reported.

To look further into the role of ethylene signalling in the two components of the root response to ABA, seedlings of three ethylene-insensitive mutants (etr1-1, ein2-1, and ein3-1) were treated with a range of concentrations of ABA. Two-way ANOVA showed that the primary root elongation rate was affected by genotype, ABA treatment and their interaction in the first $24 \mathrm{~h}$ and 4 days after treatment $(P<0.0001$, Supplementary Table 2$)$. Thus, the four genotypes responded to those ABA treatments differently and the effect of those two factors depend on each other. Figure 3 displays the effect of ABA treatment in each genotype, while Supplementary Figure 1 shows the effect of genotype under each ABA treatment.
All three mutants to varying degrees showed a diminished response to the inhibitory effect of high [ABA] compared to the wild-type (Figure 3). This was particularly evident in etr1-1 and ein2-1 during the 1st day of treatment when even the highest concentration of ABA $(30 \mu \mathrm{M})$ had no effect on the root elongation rate, and inhibited root elongation by only $14 \%$ in etr $1-1$ and even stimulated root elongation by $6 \%$ in ein2-1 compared to $48 \%$ inhibition in the wild-type (Figures 3A,C,E). A much less pronounced effect was seen in ein3-1, where $30 \mu \mathrm{M} A B A$ inhibited root elongation by $35 \%$ over the 1 st day of treatment (Figure 3G). Over the 4-day period of treatment the inhibitory effect of the high ABA concentrations was stronger in all lines, but the same pattern of decreased sensitivity in the mutants was observed (Figures 3B,D,F,H). The low ABA concentrations $(0.1$ and $0.2 \mu \mathrm{M})$ stimulated root elongation of the wild-type by $\sim 20 \%$ in the 1st day after treatment and by $\sim 30 \%$ over the full 4 days of treatment (Figures 3A,B). Similarly, the low ABA concentrations also stimulated root elongation of the three ethylene-insensitive mutants as seen after either 1 or 4 days (Figures $3 \mathbf{C}-\mathbf{H}$ ). These results confirmed the evidence from the STS treatment (Figures 2C,D) that ethylene signalling is important for the inhibitory effect of high [ABA], but not for the stimulatory effect of low $[\mathrm{ABA}]$.

\section{Investigating the Role of Auxin Transport and Signalling in the Root Responses to ABA}

To investigate the role of auxin transport in the root responses to ABA, two auxin efflux inhibitors (NPA and TIBA) and an auxin influx inhibitor (CHPAA) were firstly employed in this study. In this experiment, the stimulatory effect of the low ABA concentration $(0.1 \mu \mathrm{M})$ was only seen after 4 days treatment and not after the 1st day (Figure 4). However, some other repeated experiments showed clear effect of low ABA concentration (data not shown). This discrepancy during the first $24 \mathrm{~h}$ may be due to changes of new batches of chemicals during this study and some other unknown factors. When seedlings were grown for 4 days in the presence of either of the auxin efflux inhibitors, the stimulatory effect of $0.1 \mu \mathrm{M}$ ABA was no longer observed (Figure 4B). However, in the presence of CHPAA this concentration of ABA still had a large positive effect $(28 \%$ stimulation over CHPAA alone, compared to $34 \%$ in the control). Thus, it can be concluded that auxin efflux is necessary for the stimulatory effect of low ABA concentrations but that there is no evidence of a role for auxin influx.

Looking at the inhibitory effect of a high ABA concentration, this was surprisingly accentuated in the presence of either of the auxin efflux inhibitors, leading to an $86-89 \%$ inhibition of root elongation after 4 days, compared to $48 \%$ inhibition with $10 \mu \mathrm{M}$ ABA alone (Figure 4B). By contrast, the auxin influx inhibitor CHPAA had the effect of reducing the inhibitory effect of $10 \mu \mathrm{M}$ ABA to 6 and $23 \%$ of CHPAA alone after 1 and 4 days of treatment respectively (Figure 4). These results indicated that auxin influx is important for the root response to high ABA concentrations and that auxin efflux may play a negative role in 

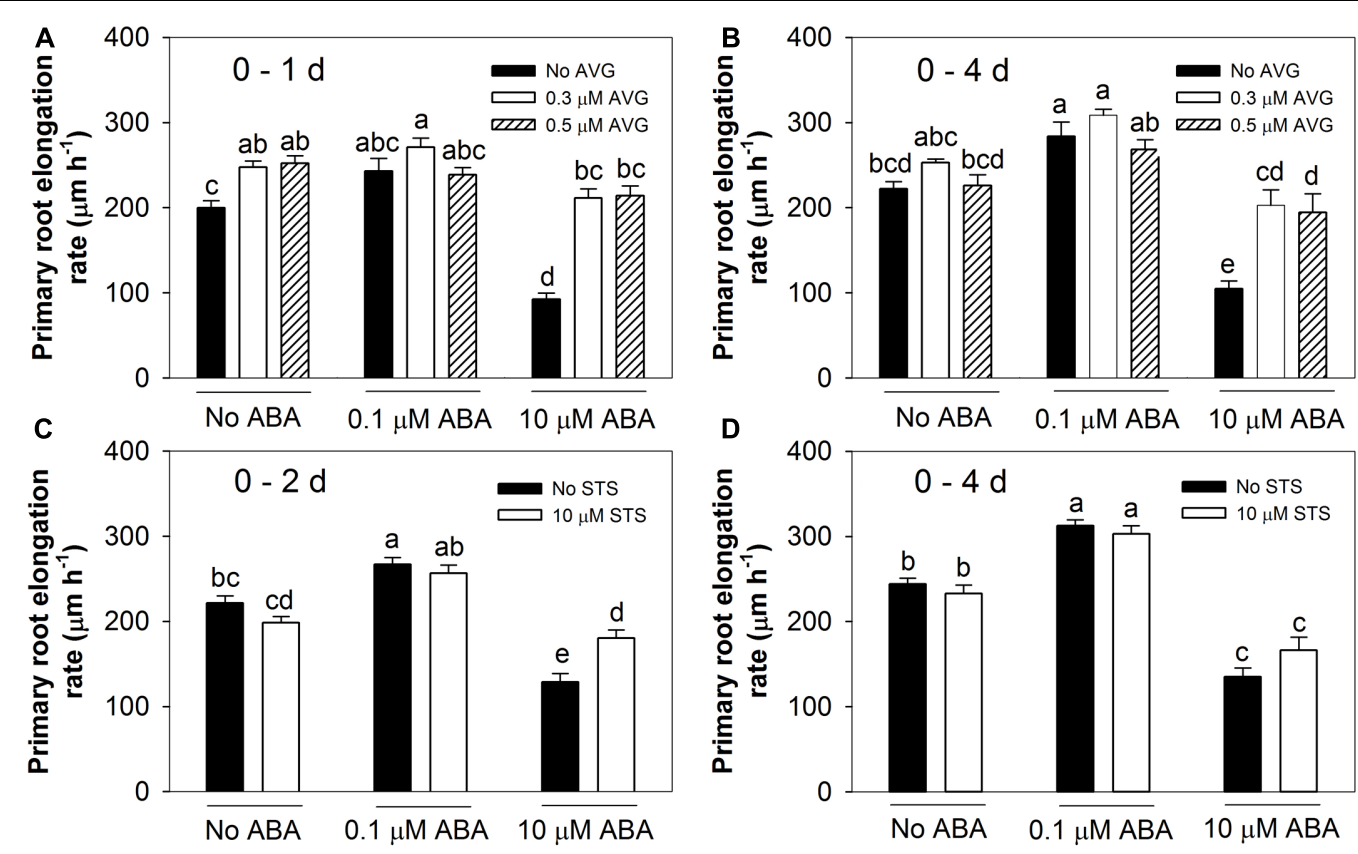

FIGURE 2 | Ethylene biosynthesis and signalling inhibitors altered root responses to ABA treatments. AVG: ethylene biosynthesis inhibitor. STS: ethylene signalling inhibitor. (A) The effects of AVG after 1 day. (B) The effects of AVG over a 4-day period. (C) The effects of STS during the first 2-day period. (D) The effects of STS during 4 days. Col-8 seedlings were germinated, chosen and transferred to medium as described in Figure 1. The medium was treated with various ABA and AVG/STS concentrations ( $\mu \mathrm{M})$. Primary root length was marked after transplanting and the increase of primary root were measured every day. The root elongation rate was calculated for the 1 or 2 days and 4 days after treatments on average. The values are means, and the vertical bars represent standard errors of the means. Data analysed using one-way ANOVA with Tukey's post hoc test and different letters indicate significant differences cross all treatments at $P<0.05$. Eta ${ }^{2}$ : (A) 0.650 ; (B) 0.622; (C) 0.729; (D) 0.817. Seedling numbers: (A) $n=14$; (B) $n=9-14$; (C) $n=9-12$; (D) $n=7-12$. At least three independent experiments were performed and similar results obtained and reported.

the mechanism by which high ABA concentrations inhibit root elongation.

A genetic approach was used to investigate the respective roles of auxin efflux and influx in the root responses to ABA. The allelic auxin influx mutants aux1-7 and aux1-T and five auxin efflux mutants (pin2/eir1-1, pin3-4, pin3-5, pin4-3, and pin7-2) were treated with a range of concentrations of ABA, and their root elongation rates were compared with that of wild-type after the 1 st day and over a 4-day period. The results of three separate experiments are shown in Figure 5. Two-way ANOVA was performed for each of those three experiments to test the impact of genotype, ABA treatment and their interaction. In all experiments, irrespective of whether measurements were made in the first $24 \mathrm{~h}$ after treatment or 4 days after treatment, there were significant effects of genotype and ABA treatment $(P<0.05$, Supplementary Table 3$)$. In the first experiment (wild-type, pin2/eir1-1, aux1-T, and iaa7/axr2-1), the results showed that there was significant genotype $\times$ ABA treatment interaction effect on the primary root elongation rate in the first $24 \mathrm{~h}$ and 4 days after treatment $(P<0.0001$, Supplementary Table 3$)$. In the second experiment (wild-type, pin4-3, pin 7-2, and tir1-1), the interaction between genotype and ABA treatment significantly affected the average primary root elongation rate after 4 days treatment $(P<0.0001)$, but not in the first $24 \mathrm{~h}$ after treatment $(P=0.42$,
Supplementary Table 3). In contrast, the results of the third experiment (wild-type, aux1-7, pin3-4, and pin 3-5) suggested that the interaction between genotype and ABA treatment significantly affected the primary root elongation rate in the first $24 \mathrm{~h}$ of $\mathrm{ABA}$ treatment $(P<0.0001)$, but not the average primary root elongation rate during the 4 days treatment $(P=0.11$, Supplementary Table 3$)$. Overall, the statistical analysis confirmed that different genotypes responded differently to ABA treatment.

In the first one of these experiments (Figures 5A,B), it was found that the aux1-T knockout mutant was insensitive to both low and high concentrations of $\mathrm{ABA}$ in the 1st day and to the higher concentration of ABA when measured over 4 days, but that a slight positive effect of low concentration of ABA could be detected after 4 days. However, the aux1-7 missense mutant showed a weaker phenotype, being unaffected in its sensitivity to low [ABA] over either 1 or 4 days (Figures 5E,F) and insensitive to high $[A B A]$ during the first $24 \mathrm{~h}$ of treatment (Figure 5E) but not during the subsequent 3 days (Figure 5F). These results are consistent with a role for AUX1-mediated auxin influx in the inhibitory effect of high [ABA], confirming the results obtained with CHPAA (Figure 4). An additional role of AUX1 in the stimulatory effect of low [ABA] cannot be ruled out but was only detectable in the early stages of treatment and only in the knockout mutant. 

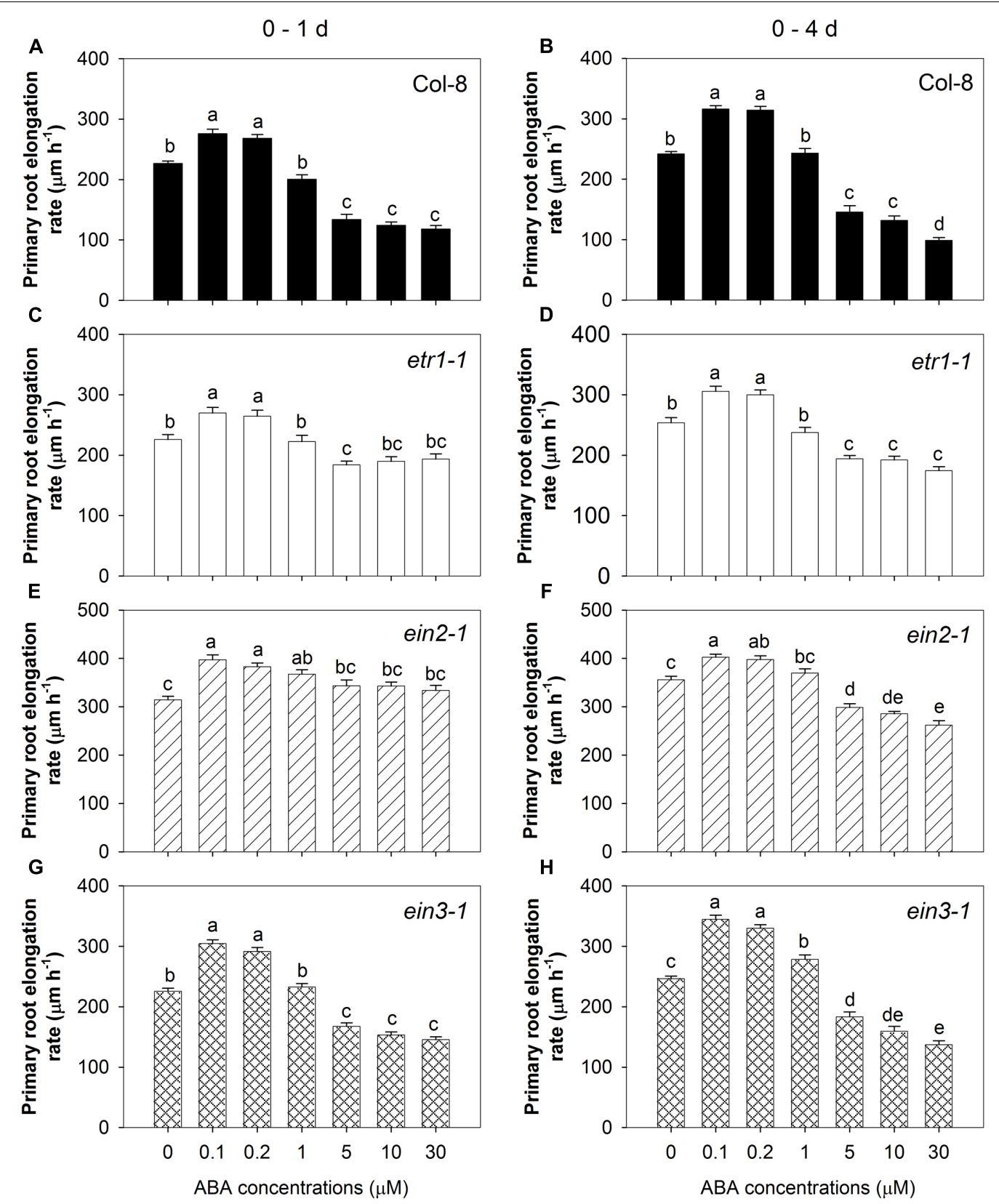

FIGURE 3 | Various responses of root growth to seven ABA treatments in three ethylene insensitive mutants and wild-type. Primary root elongation rates 1 day after treatment: (A) Col-8 wild-type; (C) etr1-1; (E) ein2-1; (G) ein3-1, and over a 4-day treatment: (B) Col-8 wild-type; (D) etr1-1; (F) ein2-1; (H) ein3-1. Seedlings of each line were germinated, chosen and transferred to medium with various ABA concentrations ( $\mu$ M) as described in Figure 1. Primary root length was marked after transplanting and the increase of primary root were measured every day. The root elongation rate was calculated for 1 and 4 days after treatments on average. Only one genotype was used in each experiment $(n=14)$, and results for each genotype came from combining two sets of independent experiments. All eight experiments were done consecutively from 17/07/2013 (day/month/year) to 26/08/2013. The values are means, and the vertical bars represent standard errors of the means. Data analysed using one-way ANOVA with Tukey's post hoc test and different letters indicate significant differences among ABA treatments at $P<0.05$. $\mathrm{Eta}^{2}$ : (A) 0.773; (B) 0.842; (C) 0.333; (D) 0.635; (E) 0.237; (F) 0.660; (G) 0.810; (H) 0.827. Seedling numbers: (A) $n=28$; (B) $n=21-28$; (C) $n=28$; (D) $n=22-28$; (E) $n=28 ;(\mathbf{F}) n=21-28 ;(\mathbf{G}) n=28$; $(\mathbf{H}) n=27-28$. At least three independent experiments were performed and similar results obtained and reported.

Of the five auxin efflux mutants tested, only pin2/eir1-1 behaved differently to the wild-type, showing less sensitivity to low [ABA], but normal sensitivity to high [ABA] (Figures 5A,B). There was one exception that in one of the three repetitions of this experiment, low $[\mathrm{ABA}](0.1 \mu \mathrm{M})$ showed similar and weak stimulatory effect in root elongation in pin2/eir1-1 as in wildtype in the 4 days treatment (by $15 \%$ vs. $17 \%$ in wild-type, data not shown). However, pin3-4, pin3-5, pin4-3, pin7-2 all showed 

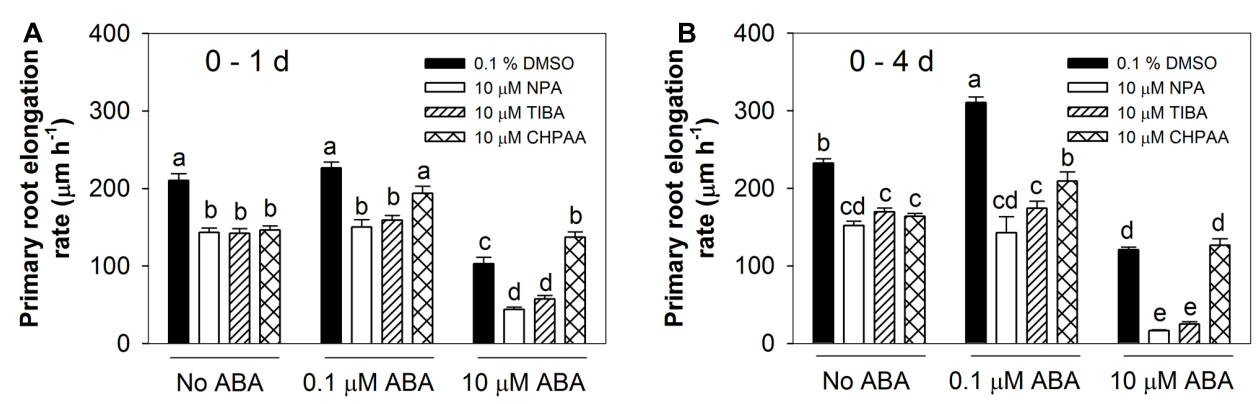

FIGURE 4 | Auxin influx and efflux inhibitors altered root responses to ABA. NPA, N-1-naphthylphthalamidic acid, auxin efflux inhibitor; TIBA, 2,3,5-triiodobenzoic acid, auxin efflux inhibitor; CHPAA, 3-chloro-4-hydroxyphenylacetic acid, auxin influx inhibitor. Primary root elongation rates (A) 1 day after treatment and (B) over a 4-day treatment period. Col-8 seedlings were germinated, chosen and transferred to medium as described in Figure 1. The medium was treated with various ABA concentrations and $0.1 \%$ DMSO or $10 \mu \mathrm{M}$ NPATIBA/CHPAA. Primary root length was marked after transplanting and the increase of primary root were measured every day. The root elongation rate was calculated for 1 day and over a 4-day treatment on average. The values are means, and the vertical bars represent standard errors of the means. Data analysed using one-way ANOVA with Tukey's post hoc test and different letters indicate significant differences cross all treatments at $P<0.05$. Eta 2 : (A) 0.834; (B) 0.951. Seedling numbers: (A) $n=10-12$; (B) $n=3-12$. At least three independent experiments were performed and similar results obtained and reported.

similar ABA responses to the wild-type (Figures 5C-F). These results are consistent with the evidence from the auxin efflux inhibitors (NPA and TIBA) that blocking auxin efflux did not alleviate the inhibitory effect of high [ABA] (Figure 4). It also suggested that the role for auxin efflux in the stimulatory effect of low $[\mathrm{ABA}]$ indicated by use of these inhibitors might involve PIN2/EIR1.

Two auxin insensitive mutants (tir1-1 and iaa7/axr2-1) were used to investigate the role of auxin signalling in the root responses to ABA. While the iaa7/axr2-1 mutant showed reduced sensitivity to both low and high [ABA] (Figures 5A,B), the tir1-1 mutant did not respond differently from the wild-type (Figures 5C,D).

\section{DISCUSSION}

\section{The Positive and Negative Effects of ABA on Root Growth Differ in Their Requirement for Ethylene Signalling}

Previous studies identified the importance of ethylene signalling and ethylene biosynthesis for the inhibition of primary root growth by ABA (Ghassemian et al., 2000; Luo et al., 2014). In the present study the objective was to try to understand how different concentrations of exogenous ABA can have opposing effects on root growth and to compare the signalling mechanisms responsible for the positive and negative responses. Use of the ethylene perception inhibitor STS (Figures 2C,D) along with three ethylene-insensitive mutants (etr1-1, ein2-1, and ein3-1) (Figure 3) confirmed that ethylene signalling was important for the inhibitory effect of high [ABA] under our experimental conditions.

In this study, the ability of the ethylene biosynthesis inhibitor AVG to completely suppress the inhibitory effect of high [ABA] (Figures 2A,B) is consistent with recent evidence that ABA inhibits root growth in Arabidopsis by promoting ethylene biosynthesis (Luo et al., 2014). The discrepancy between the present results and an earlier finding (Ghassemian et al., 2000) that AVG did not overcome the inhibitory effect of high [ABA], and even increased the degree of inhibition, could be attributable to the earlier authors' use of higher concentrations of AVG than those used here. These higher concentrations may themselves have been inhibitory to root growth through AVG's reported effects on auxin biosynthesis (Soeno et al., 2010). A recent report by Rowe et al. (2016) suggested that severe osmotic stress $(-1.2 \mathrm{MPa})$ inhibited Arabidopsis root growth independently of either ABA or ethylene. However, in their experimental system, they did not observe the promotion of root growth by either low exogenous ABA or moderate osmotic stress $(-0.37 \mathrm{MPa})$ that have been reported here and in other systems (Creelman et al., 1990; Xu et al., 2013; Rowe et al., 2016). Some other studies have also suggested that ABA and ethylene can antagonise each other and that ethylene can prevent ABA accumulation or modulate cellular sensitivity to ABA under stress (Wilkinson and Davies, 2010; Wilkinson et al., 2012; Chen et al., 2013). Therefore, the interaction between $\mathrm{ABA}$ and ethylene in stressed plants can be complex.

In contrast to the ethylene-dependence of the inhibitory effect of high [ABA], there was no evidence of any involvement of ethylene biosynthesis or signalling in the stimulatory effect of low [ABA]: neither AVG nor STS blocked the stimulatory effect (Figure 2) and the ethylene-insensitive mutants still responded positively to low [ABA] (Figure 3). These results indicate that the opposing effects of high and low ABA concentrations on root elongation operate through different signalling pathways.

Among the three ethylene-insensitive mutants tested here, the order of the effect on the root response to high [ABA] is ein2$1>\operatorname{etr} 1-1>$ ein $3-1$. These results were consistent with the report that ein 2-1 has stronger impairment in ethylene responsiveness than etr1-1 does (van Loon et al., 2006). In addition, the mutant alleles of ein 3 were previously reported to be less insensitive to ethylene than the strong alleles of etr1 and ein2 (Roman et al., 1995; Chao et al., 1997). Six members of the EIN3 family have 

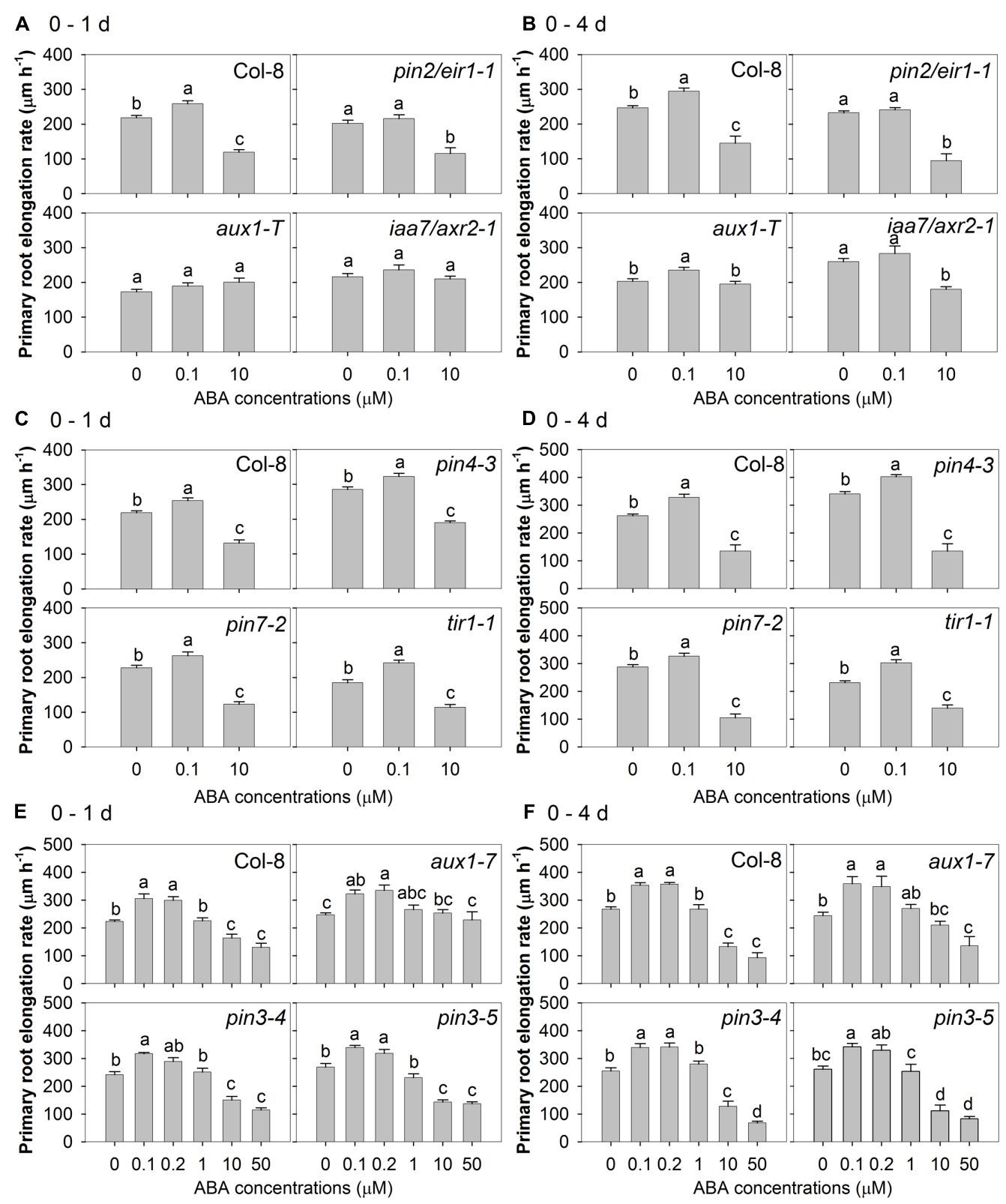

FIGURE 5 | Auxin relevant mutants showed both auxin signalling and auxin transport are required for root growth response to ABA treatments. Primary root elongation rate after the first $24 \mathrm{~h}$ treatment of: (A) wild-type Col-8, pin2/eir1-1, aux1-T, iaa7/axr2-1 (eta ${ }^{2}$ of one-way ANOVA: 0.835, 0.517, 0.118, 0.087); (C) Col-8, pin4-3, pin7-2, tir1-1 (eta ${ }^{2}$ of one-way ANOVA: 0.798, 0.840, 0.808, 0.798); (E) Col-8, aux1-7, pin3-4, pin3-5 (eta ${ }^{2}$ of one-way ANOVA: 0.773, 0.419, $0.854,0.881$ ). Average primary root elongation rate over the 4-day treatment of: (B) Col-8, pin2/eir1-1, aux1-T, iaa7/axr2-1 (eta 2 of one-way ANOVA: 0.740, 0.724, 0.324, 0.616); (D) Col-8, pin4-3, pin7-2, tir1-1 (eta ${ }^{2}$ of one-way ANOVA: 0.790, 0.884, 0.883, 0.828); (F) Col-8, aux1-7, pin3-4, pin3-5 (eta 2 of one-way ANOVA: $0.908,0.705,0.906,0.826)$. A-F were results from three experiments separately. In each experiment, seedlings of each line were germinated, chosen and transferred to medium with various ABA concentrations $(\mu \mathrm{M})$ as described in Figure 1. Primary root length was marked after transplanting and the increase of primary root were measured every day. The root elongation rate was calculated for 1 and 4 days after treatments on average. The values are means, and the vertical bars represent standard errors of the means. Data analysed using one-way ANOVA with Tukey's post hoc test and different letters indicate significant differences among ABA treatments in each genotype at $P<0.05$. Seedling numbers: (A) $n=12$; (B) $n=6-12 ;$ (C) $n=12$; (D) $n=3-12 ;(\mathbf{E}) n=8$; (F) $n=4-8$. Similar experiments were done for at least three times with different mutant combinations and similar results were obtrained.

been identified, with EIL1 being one the most closely related to EIN3 (Alonso et al., 2003b). A completely ethylene-insensitive phenotype has been reported in an ein3 eill double mutant, while the ein 3 and eill single mutants have incomplete ethylene insensitivity (Alonso et al., 2003a,b). This indicates that there is partial redundancy of function between EIN3 and EIL1, which would provide an explanation for the weaker phenotype of the ein3-1 mutant in our experiments. 


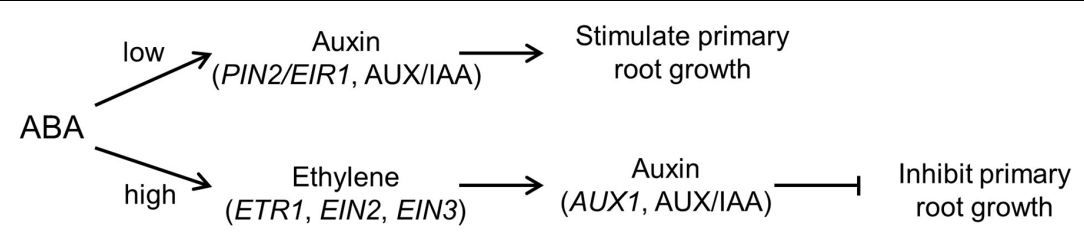

FIGURE 6 | A model for the involvement of ethylene and auxin in root growth responses to different ABA treatments. In the model, ABA regulates root growth through two distinct pathways: (1) an ethylene-independent stimulatory pathway that operates at low [ABA] and requires auxin signalling and auxin efflux through PIN2/EIR1; and (2) an ethylene-dependent inhibitory pathway that operates at high [ABA] and that also requires auxin signalling and auxin influx through AUX1. Ethylene regulates root growth through downstream auxin is based on the report that aux1-T mutant exhibited ACC-resistant root growth (Růžička et al., 2007).

\section{Auxin Signalling Is Involved in Both the Positive and Negative Responses to Exogenous ABA}

A role for auxin in the inhibitory effect of high [ABA] on Arabidopsis root growth has already been established from a number of studies using mutants defective in auxin transport and signalling (Belin et al., 2009; Wang et al., 2011; Thole et al., 2014; Zhao et al., 2015). Additionally, the distribution of GFP in the root tip of the DR5::GFP auxin reporter line was altered by adding either low or high [ABA] compared with the control (Supplementary Figure 2), which is consistent with the idea that auxin is involved in regulating root growth responses to both low and high [ABA]. However, similar distribution of GFP was observed for both low and high [ABA] treatments. These results suggest that auxin may not involve in root growth responses to low and high $[\mathrm{ABA}]$ through changing auxin maximum pattern in root tip but other means, e.g., plant sensitivity to auxin. In addition, the GFP expression patterns observed in this study were 3 days after the start of treatment, so we cannot rule out the possibility that there were short-term differences in the effects of the high and low [ABA] treatments on DR5::GFP expression that were missed in these experiments.

To look further into the involvement of auxin signalling in the root responses to both low and high [ABA], two mutants defective in components of the auxin signalling were used. The finding that iaa7/axr2-1 had reduced sensitivity to both the inhibitory effect of $10 \mu \mathrm{M}$ ABA and the stimulatory effect of $0.1 \mu \mathrm{M}$ ABA (Figures 5A,B) indicates that both the low and high [ABA] responses of root growth depend on auxin signalling. It has previously been shown that $\mathrm{ABA}$ represses the expression of the IAA7/AXR2 gene independent of auxin, leading to the suggestion that IAAT/AXR2 is at the nexus of crosstalk between $\mathrm{ABA}$ and auxin signalling pathways by acting as a negative regulator of both pathways (Belin et al., 2009). On the other hand, the gain-of-function mutant iaa7/axr2-1 has an amino acid substitution in the conserved domain II of IAA7/AXR2, which results in the accumulation of this AUX/IAA family protein and leads to repression of a series of downstream auxin responses (Nagpal et al., 2000; Gray et al., 2001). Thus, it may be that downstream components of the auxin signalling pathway, rather than IAA7/AXR2 per se, are required for both low and high [ABA] effects on root growth. The lack of a similar phenotype in another auxin signalling mutant tir1-1 (Figures 5C,D) is consistent with an earlier report that the tir1-1 mutant showed normal repression of embryonic axis elongation in response to ABA (Belin et al., 2009). This could indicate that either other F-box proteins are involved or it could be explained by genetic redundancy amongst members of this small family of auxin receptors (Dharmasiri et al., 2005; Parry et al., 2009).

\section{Differences between the Positive and Negative Responses to ABA in Their Requirements for Auxin Influx and Efflux}

Two previous studies reported that auxl auxin influx mutants were less sensitive to high concentrations of ABA than wildtype (Belin et al., 2009; Thole et al., 2014) and a pin2 auxin efflux mutant was also found to be insensitive to ABA-dependent repression of both hypocotyl and radicle elongation (Belin et al., 2009). The insensitive phenotype with respect to high [ABA] in aux 1 was confirmed in the present study (Figures 5A,B,E,F) and the role for auxin influx in ABA's inhibitory effect on root growth was further supported by the ability of the auxin influx inhibitor CHPAA to antagonise this response to high [ABA] (Figure 4). Previously, Rowe et al. (2016) found that root growth in the aux17 mutant responded similarly to the wild-type under moderate and severe osmotic stresses. However, they collected root length data only after 4 days of osmotic stress (between 5 and 9 days after germination), which would not necessarily reveal the very early ABA response of $a u x 1-7$ as shown in Figures 5E,F.

How aux 1 mutations affect the stimulatory effect of low [ABA] in our study was less clear-cut: an absence of stimulation of root growth by low [ABA] was only observed in the aux1-T knockout mutant during the first $24 \mathrm{~h}$ of treatment (Figures 5A,B). The wild-type phenotype was seen in the aux1-7 missense mutant with low [ABA] treatments (Figures 5E,F). Nevertheless, CHPAA failed to block the stimulatory effect of low [ABA] (Figure 4), indicating that there are differences between the positive and negative responses to ABA in their requirement for auxin influx.

When the positive and negative responses to $\mathrm{ABA}$ were compared for their requirement for auxin efflux, a distinct difference was found. No evidence of a positive role for auxin efflux in the inhibitory effect of ABA was obtained, based on the phenotypes of the pin2/eir1-1, pin3-4, pin3-5, pin4-3, and pin7-2 mutants (Figures 5A-F) and the inability of two auxin efflux inhibitors (NPA and TIBA) to overcome the inhibitory effect (Figure 4). On the other hand, the enhanced degree of inhibition by $10 \mu \mathrm{M} A B A$ that was seen in the presence of either NPA or TIBA in the latter experiment suggests that auxin efflux 
may play a role in counteracting the inhibitory effect of high [ABA]. PIN1 is an important auxin efflux carrier that regulates polar auxin transport and supports auxin transport from shoot to root (Gälweiler et al., 1998; Růžička et al., 2007). Reduced PIN1 expression can normally cause auxin deficiency in roots, which is accompanied by growth inhibition (Blilou et al., 2005; FernándezMarcos et al., 2011). Application of high [ABA] $(10 \mu \mathrm{M})$ to well-watered plants reduced PIN1 expression in the root tip (Rowe et al., 2016). Thus, the application of an auxin efflux inhibitor (NPA or TIBA) in combination with the high [ABA] treatment under well-watered conditions is likely to result in even lower auxin levels in root tips and much stronger inhibition of root growth. By contrast, both NPA and TIBA were successful in blocking the stimulatory effect of low [ABA] and the pin2/eir1-1 mutant (but not the other tested pin mutants) was also defective in its response to low [ABA] (Figures 4, 5A,B). This evidence of the importance of auxin efflux in the response to low [ABA] agrees with a previous report that TIBA was able to partially suppress the positive effect of a low concentration of ABA on root growth in rice (Zhao et al., 2015).

Of the four PIN genes of which the role in ABA responses was tested (PIN2, PIN3, PIN4, and PIN7), it is notable that PIN2 is the only one that is expressed in the lateral root cap (Blilou et al., 2005; Kleine-Vehn et al., 2010; Band et al., 2014). The pin2/eir1-1 mutant also shows an altered pattern of distribution of the auxin maximum in the root tip compared to other pin mutants (Ottenschläger et al., 2003; Blilou et al., 2005). It is possible that the reduced sensitivity to low [ABA] that is seen in the pin2/eir1-1 mutant might be related to specific alterations in auxin distribution that arise from loss of PIN2/EIR1's contribution to auxin efflux in the lateral root cap. On the other hand, auxin influx and efflux carriers act redundantly and a single mutation may have limited effect to ABA treatments. For example, PIN3 in the columella cells is proved to be important for gravity-sensing, but pin3 mutant showed marginal defect in gravity response, which is related to the redundancy of PIN3, PIN4, and PIN7 (Blilou et al., 2005; Kleine-Vehn et al., 2010).

The results in this study provide evidence that the stimulatory effect of low ABA concentrations on root growth operates through an ethylene-independent pathway, and requires auxin signalling and auxin transport by the PIN2/EIR1 auxin efflux carrier (Figure 6). However, the inhibitory effect seen at high ABA concentrations is through an ethylene-dependent pathway that requires auxin signalling and auxin influx through AUX1

\section{REFERENCES}

Alarcón, M. V., Lloret-Salamanca, A., Lloret, P. G., Iglesias, D. J., Talón, M., and Salguero, J. (2009). Effects of antagonists and inhibitors of ethylene biosynthesis on maize root elongation. Plant Signal. Behav. 4, 1154-1156. doi: 10.4161/psb. 4.12.9948

Alonso, J. M., Stepanova, A. N., Leisse, T. J., Kim, C. J., Chen, H., Shinn, P., et al. (2003a). Genome-wide insertional mutagenesis of Arabidopsis thaliana. Science 301, 653-657. doi: 10.1126/science.1086391

Alonso, J. M., Stepanova, A. N., Solano, R., Wisman, E., Ferrari, S., Ausubel, F. M., et al. (2003b). Five components of the ethylene-response pathway identified in
(Figure 6). Růžička et al. (2007) found ethylene inhibits root elongation by stimulating auxin biosynthesis and auxin transport toward the root elongation zone. Ethylene failed to activate auxin response and inhibit root growth in mutants that are defective in auxin perception or basipetal auxin transport (i.e., tir1-1, aux1T) (Růžička et al., 2007). In future it will be important to test the hypothesis that the same distinct pathways also account for the analogous biphasic response of root growth to different degrees of water deficit (Sharp and Davies, 1979; van der Weele et al., 2000; Xu et al., 2013). The resulting insight into how root growth responds to different degrees of soil drying could have agronomic significance in helping to manage crop development and to develop crop varieties whose root responses to soil drying are more beneficial to crop productivity in a changing climate and an increasingly water-scarce world.

\section{AUTHOR CONTRIBUTIONS}

$\mathrm{XL}, \mathrm{LC}, \mathrm{BF}$, and WD designed all of the experiments. XL performed all the lab work and data analysis. XL, LC, BF, and WD wrote the manuscript.

\section{FUNDING}

This work was supported by the EU FP7-KBBE-2011-5 project EURoot, the China Scholarship Council (CSC) and the Science Bridge Project EP/G042683/1.

\section{ACKNOWLEDGMENTS}

This paper is based on the fourth chapter of XL Ph.D. thesis at Lancaster University entitled "Plant Root Development and Hormone Signalling during Drought Stress" (2016). The authors thank Ms. Debra Hurst for her helpful support on confocal microscopy work, and Dr. Geoff Holroyd, Dr. Dawn Worrall, and Lucas Gent for their help with some of the experimental work.

\section{SUPPLEMENTARY MATERIAL}

The Supplementary Material for this article can be found online at: http://journal.frontiersin.org/article/10.3389/fpls.2017.01493/ full\#supplementary-material

a screen for weak ethylene-insensitive mutants in Arabidopsis. Proc. Natl. Acad. Sci. U.S.A. 100, 2992-2997. doi: 10.1073/pnas.0438070100

Band, L. R., Wells, D. M., Fozard, J. A., Ghetiu, T., French, A. P., Pound, M. P., et al. (2014). Systems analysis of auxin transport in the Arabidopsis root apex. Plant Cell 26, 862-875. doi: 10.1105/tpc.113. 119495

Beaudoin, N., Serizet, C., Gosti, F., and Giraudat, J. (2000). Interactions between abscisic acid and ethylene signaling cascades. Plant Cell 12, 1103-1115. doi: 10.1105/tpc.12.7.1103

Belin, C., Megies, C., Hauserova, E., and Lopez-Molina, L. (2009). Abscisic acid represses growth of the Arabidopsis embryonic axis after germination by 
enhancing auxin signaling. Plant Cell 21, 2253-2268. doi: 10.1105/tpc.109. 067702

Bensen, R. J., Boyer, J. S., and Mullet, J. E. (1988). Water deficit-induced changes in abscisic-acid, growth, polysomes, and translatable RNA in soybean hypocotyls. Plant Physiol. 88, 289-294. doi: 10.1104/pp.88.2.289

Bianchi, M. W., Damerval, C., and Vartanian, N. (2002). Identification of proteins regulated by cross-talk between drought and hormone pathways in Arabidopsis wild-type and auxin-insensitive mutants, axr1 and axf2. Funct. Plant Biol. 29, 55-61. doi: 10.1071/PP01113

Bleecker, A. B., Estelle, M. A., Somerville, C., and Kende, H. (1988). Insensitivity to ethylene conferred by a dominant mutation in Arabidopsis thaliana. Science 241, 1086-1089. doi: 10.1126/science.241.4869.1086

Blilou, I., Xu, J., Wildwater, M., Willemsen, V., Paponov, I., Friml, J., et al. (2005). The PIN auxin efflux facilitator network controls growth and patterning in Arabidopsis roots. Nature 433, 39-44. doi: 10.1038/nature03184

Chao, Q. M., Rothenberg, M., Solano, R., Roman, G., Terzaghi, W., and Ecker, J. R. (1997). Activation of the ethylene gas response pathway in Arabidopsis by the nuclear protein ETHYLENE-INSENSITIVE3 and related proteins. Cell 89, 1133-1144. doi: 10.1016/S0092-8674(00)80300- 1

Chen, L., Dodd, I. C., Davies, W. J., and Wilkinson, S. (2013). Ethylene limits abscisic acid- or soil drying-induced stomatal closure in aged wheat leaves. Plant Cell Environ. 36, 1850-1859. doi: 10.1111/pce.12094

Cramer, G. R., Urano, K., Delrot, S., Pezzotti, M., and Shinozaki, K. (2011). Effects of abiotic stress on plants: a systems biology perspective. BMC Plant Biol. 11:163. doi: 10.1186/1471-2229-11-163

Creelman, R. A., Mason, H. S., Bensen, R. J., Boyer, J. S., and Mullet, J. E. (1990). Water deficit and abscisic-acid cause differential inhibition of shoot versus root-growth in soybean seedlings - analysis of growth, sugar accumulation, and gene-expression. Plant Physiol. 92, 205-214. doi: 10.1104/pp.92. 1.205

Cutler, S. R., Rodriguez, P. L., Finkelstein, R. R., and Abrams, S. R. (2010). Abscisic acid: emergence of a core signaling network. Annu. Rev. Plant Biol. 61, 651-679. doi: 10.1146/annurev-arplant-042809-112122

Davies, P. J. (2010). "The plant hormones: their nature, occurrence, and functions," in Plant Hormones, Revised, 3rd Edn, ed. P. J. Davies (Dordrecht: Springer), 1-15. doi: 10.1007/978-1-4020-2686-7

Dharmasiri, N., Dharmasiri, S., Weijers, D., Lechner, E., Yamada, M., Hobbie, L., et al. (2005). Plant development is regulated by a family of auxin receptor F box proteins. Dev. Cell 9, 109-119. doi: 10.1016/j.devcel.2005.05.014

Fernández-Marcos, M., Sanz, L., Lewis, D. R., Muday, G. K., and Lorenzo, O. (2011). Nitric oxide causes root apical meristem defects and growth inhibition while reducing PIN-FORMED 1 (PIN1)-dependent acropetal auxin transport. Proc. Natl. Acad. Sci. U.S.A. 108, 18506-18511. doi: 10.1073/pnas.11086 44108

Gälweiler, L., Guan, C., Müller, A., Wisman, E., Mendgen, K., Yephremov, A., et al. (1998). Regulation of polar auxin transport by AtPIN1 in Arabidopsis vascular tissue. Science 282, 2226-2230. doi: 10.1126/science.282.5397.2226

Ghassemian, M., Nambara, E., Cutler, S., Kawaide, H., Kamiya, Y., and Mccourt, P. (2000). Regulation of abscisic acid signaling by the ethylene response pathway in Arabidopsis. Plant Cell 12, 1117-1126. doi: 10.1105/tpc.12.7.1117

Gray, W. M., Kepinski, S., Rouse, D., Leyser, O., and Estelle, M. (2001). Auxin regulates SCFTIR1-dependent degradation of AUX/IAA proteins. Nature 414, 271-276. doi: 10.1038/35104500

Guzmán, P., and Ecker, J. R. (1990). Exploiting the triple response of Arabidopsis to identify ethylene-related mutants. Plant Cell 2, 513-523. doi: 10.1105/tpc.2. 6.513

Hammer, G. L., Dong, Z., Mclean, G., Doherty, A., Messina, C., Schussler, J., et al. (2009). Can changes in canopy and/or root system architecture explain historical maize yield trends in the U.S. corn belt? Crop Sci. 49, 299-312. doi: $10.2135 /$ cropsci2008.03.0152

Hodge, A. (2010). Roots: the acquisition of water and nutrients from the heterogeneous soil environment. Prog. Bot. 71, 307-337. doi: 10.1007/978-3642-02167-1_12

Hodge, A., Berta, G., Doussan, C., Merchan, F., and Crespi, M. (2009). Plant root growth, architecture and function. Plant Soil 321, 153-187. doi: 10.1007/ s11104-009-9929-9

Hsiao, T. C. (1973). Plant responses to water stress. Annu. Rev. Plant Physiol. 24, 519-570. doi: 10.1146/annurev.pp.24.060173.002511
Jia, W., Wang, Y., Zhang, S., and Zhang, J. (2002). Salt-stress-induced ABA accumulation is more sensitively triggered in roots than in shoots. J. Exp. Bot. 53, 2201-2206. doi: 10.1093/jxb/erf079

Kleine-Vehn, J., Ding, Z. J., Jones, A. R., Tasaka, M., Morita, M. T., and Friml, J. (2010). Gravity-induced PIN transcytosis for polarization of auxin fluxes in gravity-sensing root cells. Proc. Natl. Acad. Sci. U.S.A. 107, 22344-22349. doi: 10.1073/pnas. 1013145107

Le, J., Vandenbussche, F., Van Der Straeten, D., and Verbelen, J. P. (2001). In the early response of Arabidopsis roots to ethylene, cell elongation is up- and downregulated and uncoupled from differentiation. Plant Physiol. 125, 519-522. doi: 10.1104/pp.125.2.519

Luo, X., Chen, Z., Gao, J., and Gong, Z. (2014). Abscisic acid inhibits root growth in Arabidopsis through ethylene biosynthesis. Plant J. 79, 44-55. doi: 10.1111/ tpj. 12534

Morgan, P. W., and Drew, M. C. (1997). Ethylene and plant responses to stress. Physiol. Plant. 100, 620-630. doi: 10.1111/j.1399-3054.1997.tb03068.x

Nagpal, P., Walker, L. M., Young, J. C., Sonawala, A., Timpte, C., Estelle, M., et al. (2000). AXR2 encodes a member of the Aux/IAA protein family. Plant Physiol. 123, 563-574. doi: 10.1104/pp.123.2.563

Ottenschläger, I., Wolff, P., Wolverton, C., Bhalerao, R. P., Sandberg, G., Ishikawa, H., et al. (2003). Gravity-regulated differential auxin transport from columella to lateral root cap cells. Proc. Natl. Acad. Sci. U.S.A. 100, 2987-2991. doi: $10.1073 /$ pnas.0437936100

Parry, G., Calderon-Villalobos, L. I., Prigge, M., Peret, B., Dharmasiri, S., Itoh, H., et al. (2009). Complex regulation of the TIR1/AFB family of auxin receptors. Proc. Natl. Acad. Sci. U.S.A. 106, 22540-22545. doi: 10.1073/pnas.0911967106

Pickett, F. B., Wilson, A. K., and Estelle, M. (1990). The AUX1 mutation of Arabidopsis confers both auxin and ethylene resistance. Plant Physiol. 94, 1462-1466. doi: 10.1104/pp.94.3.1462

Richardson, J. T. E. (2011). Eta squared and partial eta squared as measures of effect size in educational research. Educ. Res. Rev. 6, 135-147. doi: 10.1016/j.edurev. 2010.12.001

Rock, C. D., and Sun, X. (2005). Crosstalk between ABA and auxin signaling pathways in roots of Arabidopsis thaliana (L.) Heynh. Planta 222, 98-106. doi: 10.1007/s00425-005-1521-9

Roman, G., Lubarsky, B., Kieber, J. J., Rothenberg, M., and Ecker, J. R. (1995). Genetic-analysis of ethylene signal transduction in Arabidopsis thaliana: five novel mutant loci integrated into a stress response pathway. Genetics 139, 1393-1409.

Rowe, J. H., Topping, J. F., Liu, J., and Lindsey, K. (2016). Abscisic acid regulates root growth under osmotic stress conditions via an interacting hormonal network with cytokinin, ethylene and auxin. New Phytol. 211, 225-239. doi: $10.1111 /$ nph.13882

Růžička, K., Ljung, K., Vanneste, S., Podhorská, R., Beeckman, T., Friml, J., et al. (2007). Ethylene regulates root growth through effects on auxin biosynthesis and transport-dependent auxin distribution. Plant Cell 19, 2197-2212. doi: $10.1105 /$ tpc.107.052126

Saab, I. N., Sharp, R. E., Pritchard, J., and Voetberg, G. S. (1990). Increased endogenous abscisic-acid maintains primary root-growth and inhibits shoot growth of maize seedlings at low water potentials. Plant Physiol. 93, 1329-1336. doi: 10.1104/pp.93.4.1329

Saini, S., Sharma, I., Kaur, N., and Pati, P. K. (2013). Auxin: a master regulator in plant root development. Plant Cell Rep. 32, 741-757. doi: 10.1007/s00299-0131430-5

Schachtman, D. P., and Goodger, J. Q. (2008). Chemical root to shoot signaling under drought. Trends Plant Sci. 13, 281-287. doi: 10.1016/j.tplants.2008.04.003

Sharp, R. E., and Davies, W. J. (1979). Solute regulation and growth by roots and shoots of water-stressed maize plants. Planta 147, 43-49. doi: 10.1007/ BF00384589

Sharp, R. E., and LeNoble, M. E. (2002). ABA, ethylene and the control of shoot and root growth under water stress. J. Exp. Bot. 53, 33-37. doi: 10.1093/jexbot/ 53.366 .33

Sharp, R. E., Wu, Y. J., Voetberg, G. S., Saab, I. N., and Lenoble, M. E. (1994). Confirmation that abscisic-acid accumulation is required for maize primary root elongation at low water potentials. J. Exp. Bot. 45, 1743-1751. doi: 10.1093/ jxb/45.Special_Issue.1743

Soeno, K., Goda, H., Ishii, T., Ogura, T., Tachikawa, T., Sasaki, E., et al. (2010). Auxin biosynthesis inhibitors, identified by a genomics-based approach, 
provide insights into auxin biosynthesis. Plant Cell Physiol. 51, 524-536. doi: $10.1093 / \mathrm{pcp} / \mathrm{pcq} 032$

Spollen, W. G., Lenoble, M. E., Samuels, T. D., Bernstein, N., and Sharp, R. E. (2000). Abscisic acid accumulation maintains maize primary root elongation at low water potentials by restricting ethylene production. Plant Physiol. 122, 967-976. doi: 10.1104/pp.122.3.967

Thole, J. M., Beisner, E. R., Liu, J., Venkova, S. V., and Strader, L. C. (2014). Abscisic acid regulates root elongation through the activities of auxin and ethylene in Arabidopsis thaliana. G3 4, 1259-1274. doi: 10.1534/g3.114. 011080

Tian, Q., and Reed, J. W. (1999). Control of auxin-regulated root development by the Arabidopsis thaliana SHY2/IAA3 gene. Development 126, 711-721.

van der Weele, C. M., Spollen, W. G., Sharp, R. E., and Baskin, T. I. (2000). Growth of Arabidopsis thaliana seedlings under water deficit studied by control of water potential in nutrient-agar media. J. Exp. Bot. 51, 1555-1562. doi: 10.1093/jexbot/51.350.1555

van Loon, L. C., Geraats, B. P. J., and Linthorst, H. J. M. (2006). Ethylene as a modulator of disease resistance in plants. Trends Plant Sci. 11, 184-191. doi: 10.1016/j.tplants.2006.02.005

Wang, L., Hua, D. P., He, J. N., Duan, Y., Chen, Z. Z., Hong, X. H., et al. (2011). Auxin Response Factor2 (ARF2) and its regulated homeodomain gene HB33 mediate abscisic acid response in Arabidopsis. PLoS Genet. 7:e1002172. doi: 10.1371/journal.pgen.1002172

Watts, S., Rodriguez, J. L., Evans, S. E., and Davies, W. J. (1981). Root and shoot growth of plants treated with abscisic-acid. Ann. Bot. 47, 595-602. doi: 10.1093/ oxfordjournals.aob.a086056

Wilkinson, S., and Davies, W. J. (2010). Drought, ozone, ABA and ethylene: new insights from cell to plant to community. Plant Cell Environ. 33, 510-525. doi: 10.1111/j.1365-3040.2009.02052.x

Wilkinson, S., Kudoyarova, G. R., Veselov, D. S., Arkhipova, T. N., and Davies, W. J. (2012). Plant hormone interactions: innovative targets for crop breeding and management. J. Exp. Bot. 63, 3499-3509. doi: 10.1093/jxb/ ers 148

Wilson, A. K., Pickett, F. B., Turner, J. C., and Estelle, M. (1990). A dominant mutation in Arabidopsis confers resistance to auxin, ethylene and abscisic acid. Mol. Gen. Genet. 222, 377-383. doi: 10.1007/BF00633843

Xu, W., Jia, L., Shi, W., Liang, J., Zhou, F., Li, Q., et al. (2013). Abscisic acid accumulation modulates auxin transport in the root tip to enhance proton secretion for maintaining root growth under moderate water stress. New Phytol. 197, 139-150. doi: 10.1111/nph.12004

Zhang, H. M., and Forde, B. G. (1998). An Arabidopsis MADS box gene that controls nutrient-induced changes in root architecture. Science 279, 407-409. doi: $10.1126 /$ science. 279.5349 .407

Zhang, J., and Davies, W. J. (1989). Abscisic-acid produced in dehydrating roots may enable the plant to measure the water status of the soil. Plant Cell Environ. 12, 73-81. doi: 10.1111/j.1365-3040.1989.tb01918.x

Zhao, F. Y., Cai, F. X., Gao, H. J., Zhang, S. Y., Wang, K., Liu, T., et al. (2015). ABA plays essential roles in regulating root growth by interacting with auxin and MAPK signaling pathways and cell-cycle machinery in rice seedlings. Plant Growth Regul. 75, 535-547. doi: 10.1007/s10725-0140017-7

Conflict of Interest Statement: The authors declare that the research was conducted in the absence of any commercial or financial relationships that could be construed as a potential conflict of interest.

Copyright (c) 2017 Li, Chen, Forde and Davies. This is an open-access article distributed under the terms of the Creative Commons Attribution License (CC BY). The use, distribution or reproduction in other forums is permitted, provided the original author(s) or licensor are credited and that the original publication in this journal is cited, in accordance with accepted academic practice. No use, distribution or reproduction is permitted which does not comply with these terms. 